\title{
Optimal stopping of McKean-Vlasov diffusions via regression on particle systems
}

\author{
Denis Belomestny* and John Schoenmakers ${ }^{\dagger}$
}

June 26, 2018

\begin{abstract}
In this paper we study optimal stopping problems for nonlinear Markov processes driven by a McKean-Vlasov SDE and aim at solving them numerically by Monte Carlo. To this end we propose a novel regression algorithm based on the corresponding particle system and prove its convergence. The proof of convergence is based on perturbation analysis of a related linear regression problem. The performance of the proposed algorithms is illustrated by a numerical example.
\end{abstract}

\section{Introduction}

Numerical solution of multidimensional optimal stopping problems remains an important and active area of research with applications in finance, operations research and control. As the underlying models are getting more and more complex, the computational issues are becoming more relevant than ever. As a matter of fact, analytic and usual finite difference methods for solving optimal stopping problems deteriorate in high-dimensional problems. Therefore attention has turned to probabilistic approaches, based on Monte Carlo based approximative dynamic programming. Historically, one of the first motivating examples was the pricing of an American (or Bermudan) put option in a Black-Scholes model. Not much later, many American options that showed up involved high dimensional underlying processes, which has led to the development of several Monte Carlo based methods in the last decades (see e.g. [1]). Pricing American derivatives, hence solving optimal stopping problems, via Monte Carlo has always been viewed as a challenging task, because it requires backward dynamic programming that seems to be incompatible with the forward structure of Monte Carlo methods. In particular much research was

\footnotetext{
${ }^{*}$ Duisburg-Essen University. Email: denis.belomestny@uni-due.de

${ }^{\dagger}$ Weierstrass Institute of Applied Mathematics. Email: schoenma@wias-berlin.de
} 
focused on the development of fast methods to compute approximations to the optimal exercise policy. The seminal paper of Longstaff and Schwartz 13 proposed to use a cross-sectional regression over a Monte Carlo sample to compute the conditional expectations involved in the dynamic programming algorithm. The key innovation in [13] was the use of the estimated conditional expectations for decision-making, rather than quantification of expected gains. This corresponds to replacing a regression problem with a classification one, see [9]. Originally designed for the setup of American option pricing, this algorithm, which we term RMC (Regression Monte Carlo), has become widely accepted in financial mathematics, insurance and dynamic programming settings. It has also been implemented in many proprietary valuation systems employed by the financial industry. The great success of RMC is due to its flexible and simple implementation as well as its strong empirical performance. Other eminent examples of fast approximation methods include the functional optimization approach of [1, the mesh method of [7, the regression-based approaches of [8], [16, 9] and [3.

In this paper we propose and study a simulation based method for solving an optimal stopping problem for nonlinear Markov processes of the McKeanVlasov type. In spite of extensive literature on stochastic particle systems corresponding to MV-SDEs, generic numerical methods for solving optimal stopping problems in this context are hard to find (to the best of our knowledge). Our study is motivated by the recent theoretical developments in control problems for MV-SDEs and recent applications of MV-SDEs in financial mathematics. In this respect we mention the recent work of 14] (see also the references therein), where a general form of the Bellman principle is derived for the optimal control problems under McKean-Vlasov dynamics.

Because of dependence of the transition kernels on the marginal distributions, nonlinear Markov processes can not be sampled like standard diffusion processes. Instead the so-called interacting particle method combined with time discretisation is used to approximate them. However, unlike the standard Monte Carlo, the simulated particles are not independent. The key result in the theory of interacting particle systems is the so-called propagation of chaos result showing that the particles fulfil a kind of law of large numbers. In particular, one can prove strong convergence of the interacting particle system to the solution of the original McKean-Vlasov equation. Here we propose a fast approximation method for optimal stopping problems related to (generally multidimensional) MV- SDEs in spirit of the celebrated RMC method by Longstaff and Schwartz, which is based on the underlying particle system of essentially dependent particles, rather than a Monte Carlo sample of independent trajectories as in 13. In this respect one can speak about the Particle-Regression-Monte-Carlo (PRMC) method. The convergence of this method is proved via a perturbation analysis of a related linear regression problem due to an i.i.d. sample of the original MV$\mathrm{SDE}$, and a recursive error analysis of the backward induction algorithm (in the spirit of [4] and [18] for the case of independent samples). From a mathematical point of view, this analysis may be considered as the main contribution of the present paper. Summing up, we provide a generic simulation based numeri- 
cal approach for solving optimal stopping problems with respect to a reward that depends on a process following multidimensional MV-SDE. Although this problem may be relevant in a financial context, we note that financial terminology used in this paper is merely chosen for illustrative purposes, and that the application scope of the developed methods is not restricted to finance.

The structure of the paper is as follows. The general setup for optimal stopping in a Markovian environment is presented in Section 2 . In this section we also give a concise recap of the Longstaff-Schwartz and Tsitsiklis van Roy method developed in [13] and [16], respectively. Section 3 introduces MckeanVlasov equations and their connection with particle systems, while Section 4 introduces a particle version of regression based backward dynamic programming in the spirit of 13 . In Section 5 we present one of our main results, Theorem 4, that deals with the convergence of the regression approach applied to (generally dependent) particles. The convergence of the PRMC algorithm is studied in Section 6. Before proceeding to a rather general perturbation analysis in Section 8 and proving Theorem 4 and Theorem 7 in Section 9.1, we present some numerical experiments in Section 7.

\section{Optimal stopping, backward dynamic program}

Let us explain the issue of optimal stopping in the context of American options as a popular illustration. An American option grants its holder the right to select time at which she exercises the option, i.e., calls a pre-specified reward or cash-flow. This is in contrast to a European option that may be exercised only at a fixed date. A general class of American option pricing problems, i.e., optimal stopping problems respectively, can be formulated with respect to an underlying $\mathbb{R}^{d}$-valued Markov process $X:=\left\{X_{t}, 0 \leq t \leq T\right\}$ defined on a filtered probability space $\left(\Omega, \mathcal{F},\left(\mathcal{F}_{t}\right)_{0 \leq t \leq T}, \mathrm{P}\right)$. The process $X$ is assumed to be adapted to a filtration $\left(\mathcal{F}_{t}\right)_{0 \leq t \leq T}$ in the sense that each $X_{t}$ is $\mathcal{F}_{t}$ measurable. Recall that each $\mathcal{F}_{t}$ is a $\sigma$-algebra of subsets of $\Omega$ such that $\mathcal{F}_{s} \subseteq \mathcal{F}_{t}$ for $s \leq t$. In general, $X$ may describe any underlying physical or economical quantity of interest such as, for example, the outside temperature, some (not necessarily tradable) market index or price. Henceforth we restrict our attention to the case where only a finite number $\mathcal{J}$ of stopping (exercise) opportunities $0<t_{1}<t_{2}<\ldots<t_{\mathcal{J}}=T$ are allowed (Bermudan options in financial terms). In this respect it should be noted that a continuous exercise (American) option can be approximated by such a Bermudan option with arbitrary accuracy, and so this is not a huge restriction. We now consider a pre-specified reward $g_{j}\left(Z_{j}\right)$ in terms of the discrete time Markov chain

$$
Z_{i}:=X_{t_{i}}, \quad i=0, \ldots, \mathcal{J}
$$

with $Z_{0}:=X_{t_{0}}:=X_{0}$, for some given functions $g_{1}, \ldots, g_{\mathcal{J}}$ mapping $\mathbb{R}^{d}$ into $[0, \infty)$. Note that for technical convenience and without loss of generality we exclude $t_{0}=0$ from the set of exercise dates. In a financial context we may assume that the reward $g_{j}\left(Z_{j}\right)$ is expressed in units of some (tradable) pricing 
numéraire that has initial value 1 Euro, say. That is, if exercised at time $t_{j}, j=$ $1, \ldots, \mathcal{J}$, the option pays cash equivalent to $g_{j}\left(Z_{j}\right)$ units of the numéraire. Let $\mathcal{T}_{j}$ denote for $j=0, \ldots, \mathcal{J}$ the set of stopping times taking values in $\{j, j+$ $1, \ldots, \mathcal{J}\} \backslash\{0\}$. In the spirit of contingent claim pricing in finance we then assume that, for $j=0, \ldots, \mathcal{J}$, a fair price $V_{j}^{*}(z)$ of the corresponding Bermudan option at time $t_{j}$ in state $z$, given that the option was not exercised prior to $t_{j}$, is its value under the optimal exercise policy,

$$
V_{j}^{*}(z)=\sup _{\tau \in \mathcal{T}_{j}} \mathrm{E}\left[g_{\tau}\left(Z_{\tau}\right) \mid Z_{j}=z\right]=\mathrm{E}\left[g_{\tau_{j}^{*}}\left(Z_{\tau_{j}^{*}}\right) \mid Z_{j}=z\right], \quad z \in \mathbb{R}^{d},
$$

hence the solution to an optimal stopping problem. In (11) we introduced an optimal stopping family (policy), which can be also expressed in the form

$$
\tau_{j}^{*}:=\min \left\{j \leq l \leq \mathcal{J}: g_{l}\left(Z_{l}\right) \geq C_{l}^{*}\left(Z_{l}\right)\right\},
$$

where

$$
C_{j}^{*}(z):=\mathrm{E}\left[V_{j+1}^{*}\left(Z_{j+1}\right) \mid Z_{j}=z\right], \quad j=1, \ldots, \mathcal{J}-1, \quad \text { and } \quad C_{\mathcal{J}}^{*}(z) \equiv 0
$$

are so-called continuation functions. The process $V_{j}^{*}\left(Z_{j}\right)$ is called the Snell envelope of the reward process $\left(g_{k}\left(Z_{k}\right)\right)$. Both the stopping family (2) and the set of continuation functions (3) satisfy the well-known Dynamic Programming Principle. In particular, for (2) we have the backward recursion

$$
\begin{aligned}
\tau_{\mathcal{J}}^{*} & =\mathcal{J} \\
\tau_{j}^{*} & =j 1_{\left\{g_{j}\left(Z_{j}\right) \geq C_{j}^{*}\left(Z_{j}\right)\right\}}+\tau_{j+1}^{*} 1_{\left\{g_{j}\left(Z_{j}\right)<C_{j}^{*}\left(Z_{j}\right)\right\}}, \quad j=1, \ldots, \mathcal{J}-1,
\end{aligned}
$$

and for (3) it holds,

$$
\begin{aligned}
C_{\mathcal{J}}^{*}(z) & \equiv 0 \\
C_{j}^{*}(z) & =\mathrm{E}\left[g_{\tau_{j+1}^{*}}\left(Z_{\tau_{j+1}^{*}}\right) \mid Z_{j}=z\right] \\
& =\mathrm{E}\left[\max \left(g_{j+1}\left(Z_{j+1}\right), C_{j+1}^{*}\left(Z_{j+1}\right)\right) \mid Z_{j}=z\right], \quad j=1, \ldots, \mathcal{J}-1 .
\end{aligned}
$$

A common feature of almost all existing fast approximation algorithms is that they deliver estimates $C_{N, 1}(z), \ldots, C_{N, \mathcal{J}-1}(z)$ for the continuation functions $C_{1}^{*}(z), \ldots, C_{\mathcal{J}-1}^{*}(z)$. Here the index $N$ indicates that the above estimates are based on the set of independent "training" trajectories $\left(Z_{0}^{(i)}, \ldots, Z_{\mathcal{J}}^{(i)}\right), i=$ $1, \ldots, N$, all starting from one point $Z_{0}$ i.e., $Z_{0}=Z_{0}^{(1)}=\ldots=Z_{0}^{(N)}$. In the case of regression methods the estimates for the continuation values are obtained via regression based Monte Carlo. For example, at step $\mathcal{J}-j$ one may estimate the expectation

$$
\left.\mathrm{E}\left[\max \left(g_{j+1}\left(Z_{j+1}\right), C_{N, j+1}\left(Z_{j+1}\right)\right)\right) Z_{j}=z\right]
$$

via linear regression based on the set of paths

$$
\left(Z_{j}^{(i)}, \max \left\{g_{j+1}\left(Z_{j+1}^{(i)}\right), C_{N, j+1}\left(Z_{j+1}^{(i)}\right)\right\}\right), \quad i=1, \ldots, N,
$$


where $C_{N, j+1}(z)$ is the estimate for $C_{j+1}^{*}(z)$ obtained in the previous step, and $C_{N, \mathcal{J}}(z):=0$. This approach is basically the method by Tsitsiklis and van Roy [16]. Alternatively, in the so-called Longstaff-Schwarz algorithm, one mixes the estimates of the continuation values with the corresponding estimates of the stopping times. More precisely, on each trajectory $i=1, \ldots, N$, approximate stopping times $\tau_{N, j}^{(i)}, j=1, \ldots, \mathcal{J}$, are recursively constructed by first initializing $\tau_{N, \mathcal{J}}^{(i)}=\mathcal{J}$, for all $i$. Then, once $\tau_{N, j+1}^{(i)}, i=1, \ldots, N$, is constructed for $j<\mathcal{J}$, one computes from the sample

$$
\left(Z_{j}^{(i)}, g_{\tau_{N, j+1}^{(i)}}\left(Z_{\tau_{N, j+1}^{(i)}}^{(i)}\right)\right), \quad i=1, \ldots, N
$$

an estimate $C_{N, j}(z)$ of the continuation function $C_{j+1}^{*}(z)$ by projection on the linear span of a set of basis functions. Subsequently, the approximate stopping times corresponding to exercise date $j$ on trajectories $i=1, \ldots, N$, are defined via,

$$
\tau_{N, j}^{(i)}=j 1_{\left\{g_{j}\left(Z_{j}^{(i)}\right) \geq C_{N, j}\left(Z_{j}^{(i)}\right)\right\}}+\tau_{N, j+1}^{(i)} 1_{\left\{g_{j}\left(Z_{j}^{(i)}\right)<C_{N, j}\left(Z_{j}^{(i)}\right)\right\}} \cdot
$$

Working all the way back, we thus obtain again a set of approximate continuation functions $C_{N, 1}(z), \ldots, C_{N, \mathcal{J}-1}(z)$ (note that $C_{N, \mathcal{J}}(z) \equiv 0$ ).

Remark 1 It should be noted that the algorithm based on (7) is more popular than the one based on ([6), because it behaves more stable in practice, particularly when the number of exercise dates is getting very large. Intuitively, this can be explained by the fact that the regression (7) is always carried out on actually realized cash-flows, rather than on approximative value functions as in (6) which may become quite unprecise because of error propagation due to a huge number of exercise dates, see for example [3] for more rigorous arguments.

Given the estimates $C_{N, 1}, \ldots, C_{N, \mathcal{J}-1}$, constructed by one of the fast approximation methods above, we can construct a lower bound (low biased estimate) for $V_{0}^{*}$ using the (generally suboptimal) stopping rule:

$$
\tau_{N}=\min \left\{1 \leq j \leq \mathcal{J}: g_{j}\left(Z_{j}\right) \geq C_{N, j}\left(Z_{j}\right)\right\}
$$

with $C_{N, \mathcal{J}} \equiv 0$ by definition. Indeed, fix a natural number $N_{\text {test }}$ and simulate $N_{\text {test }}$ new independent trajectories of the process $Z$. A low-biased estimate for $V_{0}^{*}$ can then be defined as

$$
V_{0}^{N_{\text {test }}, N}=\frac{1}{N_{\text {test }}} \sum_{r=1}^{N_{\text {test }}} g_{\tau_{N}^{(r)}}\left(Z_{\tau_{k}^{(r)}}^{(r)}\right)
$$

with

$$
\tau_{N}^{(r)}=\inf \left\{1 \leq j \leq \mathcal{J}: g_{j}\left(Z_{j}^{(r)}\right) \geq C_{N, j}\left(Z_{j}^{(r)}\right)\right\}, \quad r=1, \ldots, N_{\text {test }} .
$$




\section{McKean-Vlasov equations and particle systems}

Let $[0, T]$ be a finite time interval and $(\Omega, \mathcal{F}, \mathrm{P})$ be a complete probability space, where a standard $m$-dimensional Brownian motion $W$ is defined. We consider a class of McKean-Vlasov stochastic differential equations (MVSDE), i.e., stochastic differential equations whose drift and diffusion coefficients may depend on the current distribution of the process, of the form:

$$
\left\{\begin{array}{l}
X_{t}=\xi+\int_{0}^{t} \int_{\mathbb{R}^{d}} a\left(X_{s}, y\right) \mu_{s}(d y) d s+\int_{0}^{t} \int_{\mathbb{R}^{d}} b\left(X_{s}, y\right) \mu_{s}(d y) d W_{s} \\
\mu_{t}=\operatorname{Law}\left(X_{t}\right), \quad t \geq 0, \quad X_{0} \sim \mu_{0}
\end{array}\right.
$$

where $\mu_{0}$ is a distribution in $\mathbb{R}^{d}, a: \mathbb{R}^{d} \times \mathbb{R}^{d} \rightarrow \mathbb{R}^{d}$ and $b: \mathbb{R}^{d} \times \mathbb{R}^{d} \rightarrow \mathbb{R}^{d \times m}$. A popular way of simulating the MVSDE (9) is to sample the so-called particle system $\mathbf{X}_{t}^{N}=\left(X_{t}^{1, N}, \ldots, X_{t}^{N, N}\right) \in \mathbb{R}^{d \times N}$ from $N \times d$-dimensional SDE

$$
X_{t}^{i, N}=\xi^{i}+\frac{1}{N} \sum_{j=1}^{N} \int_{0}^{t} a\left(X_{s}^{i, N}, X_{s}^{j, N}\right) d s+\frac{1}{N} \sum_{j=1}^{N} \int_{0}^{t} b\left(X_{s}^{i, N}, X_{s}^{j, N}\right) d W_{s}^{i}
$$

for $i=1, \ldots, N$, where $\xi^{i}, i=1, \ldots, N$, are i.i.d copies of a r.v. $\xi$, distributed according the law $\mu_{0}$, and $W^{i}, i=1, \ldots, N$, are independent copies of $W$. Under suitable assumptions (see, for example [2]) one has that

$$
\left\|\sup _{0 \leq r \leq T}\left|X_{r}^{\cdot, N}-X_{r}\right|\right\|_{p} \leq C_{p} N^{-1 / 2} .
$$

In practice, $N \times d$-dimensional SDE system (10) cannot be solved analytically either and one has to approximate its solution by a suitable numerical integration scheme such as the Euler method, leading to a next approximation $\mathbf{X}_{t}^{N, \delta}=\left(X_{t}^{1, N, \delta}, \ldots, X_{t}^{N, N, \delta}\right)$ with $\delta$ being the size of the each Euler time step. Following [2], one then has

$$
\left\|\sup _{0 \leq r \leq T}\left|X_{r}^{\cdot, N, \delta}-X_{r}^{\cdot, N}\right|\right\|_{p} \lesssim \sqrt{\delta}
$$

where $\lesssim$ involves a constant that does not depend on $N$ and $\delta$.

Remark 2 In order to fix the main ideas and to avoid a notational blow up, we assume in this paper that the system $\mathbf{X}_{t}^{N}(c f$. (10)) is constructed exactly, hence we neglect the numerical integration error (12). On the other hand, due to (12) it will be clear how several results in this paper have to be adapted in the case where (10) is approximated using the Euler scheme.

Remark 3 In fact, the solution to the MVSDE (9) may be considered as a usual non-autonomous, Markovian diffusion, since $\left\{\mu_{s}: 0 \leq s \leq T\right\}$ is some deterministic flow of distributions, although not explicitly known beforehand. Therefore, we may consider the stopping problem (1) with respect to the solution (9), 
while the standard notions of the Snell envelope and the Dynamic Programming Principle still apply. However, in contrast to the standard diffusion processes $X$, where independent trajectories of $X$ may be simulated straightforwardly by Monte Carlo, simulating of independent copies of (9) is not directly possible. As a way out, we will work with the particle system (10) of dependent particles, instead of an ensemble of independent trajectories of (9).

\section{Dynamic programming on particle systems}

In this section we describe a particle version of the Longstaff-Schwarz regression algorithm due to (77) and (8). First we run the particle system $\mathbf{X}_{t}^{N}=$ $\left(X_{t}^{1, N}, \ldots, X_{t}^{N, N}\right) \in \mathbb{R}^{d \times N}$ as described above and set

$$
Z_{j}^{i, N}=X_{j \Delta}^{i, N}, \quad j=0, \ldots, \mathcal{J}, \quad i=1, \ldots, N,
$$

with $\mathcal{J}=\lfloor T / \Delta\rfloor$. It should be noted that, unlike Monte Carlo, the trajectories (13) are generally dependent. We now consider an approximative dynamic programming algorithm based on the (generally dependent) paths $\mathbf{Z}^{N}=\left(Z_{j}^{i, N}, i=\right.$ $1, \ldots, N, j=0, \ldots, \mathcal{J})$. In the spirit of the Longstaff-Schwarz algorithm we compute sequentially for $j=\mathcal{J}, \ldots, 1$, approximate continuation functions $C_{N, j}$ and approximate stopping times $\tau_{N, j}^{(i)}, i=1, \ldots, N$. That is, we initialize $\tau_{N, \mathcal{J}}^{(i)}=\mathcal{J}$, $i=1, \ldots, N$, and $C_{N, \mathcal{J}}=0$, and once $\tau_{N, j+1}^{(i)}, i=1, \ldots, N$, and $C_{N, j+1}, j<\mathcal{J}$, are constructed, $C_{N, j}$ is obtained from solving the minimization,

$$
C_{N, j}:=\underset{h \in \mathcal{H}_{K}}{\arg \min }\left\{\frac{1}{N} \sum_{i=1}^{N}\left(g_{\tau_{N, j+1}^{(i)}}\left(Z_{\tau_{N, j+1}^{(i)}}^{i, N}\right)-h\left(Z_{j}^{i, N}\right)\right)^{2}\right\}
$$

$\left(C_{N, \mathcal{J}}:=0\right)$. Next $\tau_{N, j}^{(i)}$ is updated analogue to the scheme (8), i.e.,

$$
\tau_{N, j}^{(i)}=j 1_{\left\{g_{j}\left(Z_{j}^{i, N}\right) \geq C_{N, j}\left(Z_{j}^{i, N}\right)\right\}}+\tau_{N, j+1} 1_{\left\{g_{j}\left(Z_{j}^{i, N}\right)<C_{N, j}\left(Z_{j}^{i, N}\right)\right\}} .
$$

Note that, for fixed $j$, the pairs

$$
\left(Z_{j}^{i, N}, g_{\tau_{N, j+1}^{(i)}}\left(Z_{\tau_{N, j+1}^{(i)}}^{i, N}\right)\right), \quad i=1, \ldots, N
$$

are generally dependent, but, have the same distribution for each $i$. As such (14) is indeed can be viewed as an estimator of $C_{j}^{*}$ (cf. (44). Usually the space $\mathcal{H}_{K}$ is taken to be the linear span of some given set of basis functions, so that the minimization (14) boils down to a linear least squares problem that can be solved via straightforward linear algebra. Let us refer to the above algorithm as the PRMC algorithm. 


\section{$5 \quad$ Regression on interacting particle systems}

In this section we consider for some fixed $t \geq 0$ and time $T>t$ a generic problem of computing the functionals of the form

$$
w(x)=\mathrm{E}\left[f\left(X_{T}\right) \mid X_{t}=x\right],
$$

where $\left(X_{t}\right)$ is the solution of (9). In (16), $T$ may in general be any random time. Let $\mathbf{X}_{t}^{N}=\left(X_{t}^{1, N}, \ldots, X_{t}^{N, N}\right)$ be a particle system (10). Furthermore, let for each $K \in \mathbb{N}, \mathcal{H}_{K}$ be a $K$-dimensional linear space of functions $h: \mathbb{R}^{d} \rightarrow \mathbb{R}$ and consider the estimate

$$
\widetilde{w}_{N}:=\underset{h \in \mathcal{H}_{K}}{\arg \min }\left\{\frac{1}{N} \sum_{i=1}^{N}\left(f\left(X_{T}^{i, N}\right)-h\left(X_{t}^{i, N}\right)\right)^{2}\right\},
$$

where the dimension $K$ may depend on $N$. Let $\left(\psi_{k}\right)_{k=1,2, \ldots}$ be a sequence of linearly independent basis functions and set $\mathcal{H}_{K}:=\operatorname{span}\left\{\psi_{1}, \ldots, \psi_{K}\right\}$. In this section we are going to analyze the properties of the estimate $\widetilde{w}_{N}$. Note that the random variables $X_{T}^{1, N}, \ldots, X_{T}^{N, N}$ are generally dependent, so that the known results from regression analysis (see, e.g. [12]) can not be applied directly. Consider the truncated version of the estimate $\widetilde{w}_{N}$ defined as $T_{M} \widetilde{w}_{N}$, where $T_{M}$ is a truncation operator defined for a generic function $h$ and a threshold $M$ as

$$
T_{M} h= \begin{cases}M, & h>M \\ h, & -M \leq f \leq M \\ -M, & h<-M\end{cases}
$$

We have the following theorem.

Theorem 4 Assume that all functions $\psi_{k}, k=1,2, \ldots$ and $f$ are globally bounded and Lipschitz continuous. That is, there exist constants $M_{f}, L_{f}, L_{k}$, $M_{k}, \ell_{k}, k=1,2, \ldots$ such that for all $x, y \in \mathbb{R}^{d}$,

$$
\begin{gathered}
|f(x)| \leq M_{f}, \quad \frac{1}{K} \sum_{k=1}^{K}\left|\psi_{k}(x)\right|^{2} \leq M_{K}^{2}, \\
\left|\psi_{k}(x)-\psi_{k}(y)\right| \leq L_{k}|x-y|, \quad|f(x)-f(y)| \leq L_{f}|x-y| .
\end{gathered}
$$

Further suppose that

$$
0<\varkappa_{\circ} \leq \lambda_{\min }\left(\Sigma_{K}\right)<\lambda_{\max }\left(\Sigma_{K}\right) \leq \varkappa^{\circ}<\infty
$$

for all $K \in \mathbb{N}$, where

$$
\Sigma_{K}=\left(\int \psi_{k}(x) \psi_{l}(x) \mu_{t}(d x), k, l=1, \ldots, K\right) .
$$


Then, it holds

$$
\begin{aligned}
& \left\|T_{M_{f}} \widetilde{w}^{N}(x)-w(x)\right\|_{L_{2}\left(\mu_{t}\right)} \\
& \begin{array}{r}
\lesssim \frac{M_{K} \sqrt{K}}{\sqrt{N}}\left[d_{1} M_{f} \ell_{K}+d_{2} L_{f}\right]+\frac{M_{f}}{\sqrt{N}}\left[d_{3} \ell_{K}+\sqrt{1+\log N} \sqrt{K}\right] \\
\quad+M_{f} \sqrt{K} \exp \left[-d_{4} \frac{N}{K M_{K}^{2}}\right]+\inf _{h \in \mathcal{H}_{K}}\|h-w\|_{L_{2}\left(\mu_{t}\right)}
\end{array}
\end{aligned}
$$

with

$$
\ell_{K}^{2}:=\sum_{k=1}^{K} L_{k}^{2} .
$$

In (22) the constants $d_{1,2,3,4}$ depend on $\varkappa_{0}, \varkappa^{\circ}$ only, $\lesssim$ denotes $\leq$ up to a universal constant for each term.

Theorem 4 will be proved in Section 9.1 .

\section{Convergence analysis of the PRMC algorithm}

In this section we investigate the convergence properties of the PRMC regression algorithm. To this end, we modify the PRMC algorithm in such a way that our fundamental result, Theorem 4 may be applied. In fact, we follow an approach in the spirit of [18] (cf. 4]), and assume that instead of one particle sample $\mathbf{Z}^{N}$, we have at hand for $j=1, \ldots, \mathcal{J}-1$, independent particle samples $\mathbf{Z}^{j ; N}:=\left(Z_{r}^{j ; i, N}, i=1, \ldots, N, r=0, \ldots, \mathcal{J}\right)$, all starting at $Z_{0}=X_{0}$. We next assume that $C_{N, j}$ and $\tau_{N, j}$ are constructed in the following backward recursive way: As initialization we set $\tau_{N, \mathcal{J}}=\mathcal{J}$ and $C_{N, \mathcal{J}} \equiv 0$. Once $C_{N, j+1}$ and $\tau_{N, j+1}$, $j+1 \leq \mathcal{J}$, are determined based on the samples $\mathbf{Z}^{j+1 ; N}, \ldots, \mathbf{Z}^{\mathcal{J}-1 ; N}$, then $C_{N, j}$ is constructed based on the samples $\mathbf{Z}^{j ; N}, \ldots, \mathbf{Z}^{\mathcal{J}-1 ; N}$, via

$$
C_{N, j}=\underset{h \in \mathcal{H}_{K}}{\arg \min } \sum_{i=1}^{N}\left(g_{\tau_{N, j+1}^{(i)}}\left(Z_{\tau_{N, j+1}^{(i)}}^{j ; i, N}\right)-h\left(Z_{j}^{j ; i, N}\right)\right)^{2},
$$

and, subsequently, $\tau_{N, j}$ is defined by

$$
\tau_{N, j}:=j 1_{\left\{g_{j}\left(Z_{j}\right) \geq C_{N, j}\left(Z_{j}\right)\right\}}+\tau_{N, j+1} 1_{\left\{g_{j}\left(Z_{j}\right)<C_{N, j}\left(Z_{j}\right)\right\}},
$$

for a generic dummy trajectory $\left(Z_{l}\right)_{l=0, \ldots, \mathcal{J}}$ corresponding to the (exact) solution of (9) independent of

$$
\mathcal{G}_{j}:=\sigma\left\{\mathbf{Z}^{j ; N}, \ldots, \mathbf{Z}^{\mathcal{J}-1 ; N}\right\}
$$

Let us further define

$$
\bar{C}_{N, j}(z):=\mathrm{E}_{\mathcal{G}_{j+1}}\left[g_{\tau_{N, j+1}}\left(Z_{\tau_{N, j+1}}\right) \mid Z_{j}=z\right] .
$$


Note that the random function $\bar{C}_{N, j}$ is $\mathcal{G}_{j+1}$-measurable while its estimate $C_{N, j}$ is $\mathcal{G}_{j}$-measurable. By running this procedure all the way down to $j=1$, we so end up with a sequence of approximative continuation functions $C_{N, j}(\cdot)$, and the corresponding conditional expectations $\bar{C}_{N, j}(z)$. The following lemma holds.

Lemma 5 For the conditional expectations 24) we have that,

$$
\left\|\bar{C}_{N, j}-C_{j}^{*}\right\|_{L_{p}\left(\mu_{j}\right)} \leq \sum_{l=j+1}^{\mathcal{J}-1}\left\|C_{N, l}-C_{l}^{*}\right\|_{L_{p}\left(\mu_{l}\right)}
$$

with $p \geq 1$ by slightly abusing notation and using $\mu_{j}=\mu_{t_{j}}$. Note that the inequality (25) involves $\mathcal{G}_{j+1}$-measurable objects.

Remark 6 It is interesting to compare the estimate (25) with similar ones in Lemma 2.3 of [18].

The following theorem states the convergence of the approximate continuation functions in the PRMC algorithm to the exact ones, respectively.

Theorem 7 Assume that the conditions (19), (20), (21) are fulfilled with $f$ replaced by $g_{j}$ uniformly in $j=1, \ldots, \mathcal{J}$. By denoting the norm

$$
\|\cdot\|_{L_{2}\left(\mu_{j}, \mathbb{P}\right)}^{2}:=\mathrm{E}\left[\|\cdot\|_{L_{2}\left(\mu_{j}\right)}^{2}\right],
$$

due to the unconditional expectation with respect to the "all in" probability measure $\mathbb{P}$, one has for $j=1, \ldots, \mathcal{J}-1$,

$$
\left\|C_{N, \mathcal{J}-j}-C_{\mathcal{J}-j}^{*}\right\|_{L_{2}\left(\mu_{\mathcal{J}-j}, \mathbb{P}\right)} \leq \Delta_{N, K}(2+c)^{j},
$$

where $\Delta_{N, K}=c\left(\epsilon_{N, K}+\max _{j} \inf _{h \in \mathcal{H}_{K}}\left\|C_{j}^{*}-h\right\|_{L_{2}\left(\mu_{j}\right)}\right)$ for some $c>0$ with

$$
\epsilon_{N, K}=\sqrt{\frac{K}{N}}\left(M_{K} \ell_{K}+\eta_{1} \sqrt{1+\log N}\right)+\eta_{2} \sqrt{K} \exp \left[-\eta_{3} \frac{N}{K M_{K}^{2}}\right]
$$

for some constants $\eta_{1,2,3}>0$ not depending on $K$ and $N$.

Example 8 The Hermite polynomial of order $j$ is given, for $j \geq 0$, by:

$$
H_{j}(x)=(-1)^{j} e^{x^{2}} \frac{d^{j}}{d x^{j}}\left(e^{-x^{2}}\right) .
$$

Hermite polynomials are orthogonal with respect to the weight function $e^{-x^{2}}$ and satisfy: $\int_{\mathbb{R}} H_{j}(x) H_{\ell}(x) e^{-x^{2}} d x=2^{j} j ! \sqrt{\pi} \delta_{j, \ell}$. The Hermite function of order $j$ is given by:

$$
\psi_{j}(x)=c_{j} H_{j}(x) e^{-x^{2} / 2}, \quad c_{j}=\left(2^{j} j ! \sqrt{\pi}\right)^{-1 / 2} .
$$

The sequence $\left(\psi_{j}, j \geq 0\right)$ is an orthonormal basis of $L_{2}(\mathbb{R})$. The infinite norm of $\psi_{j}$ satisfies (see Szegö [15] p.242):

$$
\left\|\psi_{j}\right\|_{\infty} \leq M_{0}, \quad M_{0} \simeq 1,086435 / \pi^{1 / 4} \simeq 0.8160 .
$$

Furthermore, since $\psi_{k}^{\prime}(x)=\sqrt{k / 2} \psi_{k-1}(x)-\sqrt{(k+1) / 2} \psi_{k+1}(x)$ we derive that the condition (20) is fulfilled with $L_{k}=2 M_{0} \sqrt{k}$. 


\section{Numerical experiment}

As a simple illustration of the proposed methodology, let us consider optimal stopping problem in the so-called Shimizu-Yamada model

$$
d X_{t}=\left(a \mathrm{E}\left[X_{t}\right]+b X_{t}\right) d t+\sigma d W_{t}, \quad X_{0}=x_{0}, \quad t \in[0, T]
$$

(see [10], Section 3.10), which has the explicit solution

$$
X_{t}=x_{0} e^{(a+b) t}+\sigma \int_{0}^{t} e^{b(t-s)} d W_{s}
$$

that in turn solves the ordinary SDE

$$
d X_{t}=\left(x_{0} a e^{(a+b) t}+b X_{t}\right) d t+\sigma d W_{t}
$$

(cf. [5]). It is straightforward to show that the conditional mean and variance of (30) are given by

$$
\begin{aligned}
\mathrm{E}\left[X_{t} \mid X_{s}\right] & =e^{b(t-s)} X_{s}+x_{0} e^{b t}\left(e^{a t}-e^{a s}\right) \quad \text { and } \\
\operatorname{Var}\left[X_{t} \mid X_{s}\right] & =\sigma^{2} \frac{e^{2 b(t-s)}-1}{2 b}, \quad 0 \leq s \leq t,
\end{aligned}
$$

respectively. That is, for $s=0$ we have that

$$
\mathrm{E}\left[X_{t}\right]=x_{0} e^{(a+b) t} \quad \text { and } \quad \operatorname{Var}\left[X_{t}\right]=\sigma^{2} \frac{e^{2 b t}-1}{2 b} .
$$

In the particular case $b=-a, a>0$ one so has

$$
\begin{aligned}
\mathrm{E}\left[X_{t} \mid X_{s}\right] & =e^{-a(t-s)} X_{s}+x_{0}\left(1-e^{-a(t-s)}\right), \\
\operatorname{Var}\left[X_{t} \mid X_{s}\right] & =\sigma^{2} \frac{1-e^{-2 a(t-s)}}{2 a}, \quad 0 \leq s \leq t, \quad a>0,
\end{aligned}
$$

and (32) yields $\mathrm{E}\left[X_{t}\right]=x_{0}$ for all $t$. For this case the particle system (10) reads

$$
X_{t}^{i, N}=x_{0}+\frac{a}{N} \sum_{j=1}^{N} \int_{0}^{t} X_{s}^{j, N} d s-a \int_{0}^{t} X_{s}^{i, N} d s+\sigma W_{t}^{i}, \quad t \in[0, T] .
$$

We now consider the optimal stopping problem

$$
V_{0}^{*}=\sup _{\tau \in \mathcal{T}_{j}} \mathrm{E}\left[g_{\tau}\left(X_{t_{\tau}}\right)\right]
$$

for some reward functions $g_{j}: \mathbb{R} \rightarrow \mathbb{R}_{\geq 0}, t_{j}=j T / \mathcal{J}$, where $\left(X_{t}\right)$ solves (29) with $b=-a, a>0$. Since $X$ follows an ordinary SDE (31), we may compute an approximation to (35) numerically by the Longstaff-Schwarz method 13 based on independent trajectories of (31), and then compare it to the particle based 
Longstaff-Schwarz algorithm proposed in Section 4 Let us further consider, for illustration a Bermudan put option (in financial terms),

$$
g_{j}(x)=e^{-r t_{j}}(x-K)^{+}, \quad j=0, \ldots, \mathcal{J},
$$

for some $K>0$, where $r$ can be interpreted as interest rate. We take the following parameters $d=1, x_{0}=1, K=0.1, a=1, T=1, \mathcal{J}=100$ and implement the following two phase algorithm. In the first stage we run $N_{\text {tr }}$ trajectories either of the particle system (34) or of the process (31). Using these trajectories, we estimate the corresponding continuation functions using linear regression with quadratic polynomials and reward functions as basis functions. In the second stage we use the estimated continuation values on a new set of $N_{\text {test }}=5000$ testing trajectories to construct a suboptimal stopping rule and consequently a lower bound for $V_{0}^{*}$ by averaging over the testing paths. We also compute dual upper bounds using the estimated continuation functions and $N_{\text {in }}=100$ inner paths to approximate one step conditional expectations, see, e.g. Chapter 3 in [6]. The results for different values of $N_{\text {tr }}$ are shown in Table 1 As can be seen from Table 1, the PRMC (Particle Regression MC)

\begin{tabular}{|c|c|c|}
\hline$N_{\text {tr }}$ & RMC & PRMC \\
\hline \hline 10 & {$[0.9393(0.0079), 1.2742(0.0076)]$} & {$[0.9287(0.0058), 1.1750(0.0038)]$} \\
\hline 50 & {$[1.0047(0.0082), 1.0942(0.0019)]$} & {$[0.9829(0.0072), 1.1745(0.0041)]$} \\
\hline 100 & {$[1.0144(0.0073), 1.0871(0.0013)]$} & {$[1.0079(0.0080), 1.0978(0.0023)]$} \\
\hline 300 & {$[1.0342(0.0077), 1.0718(0.0009)]$} & {$[1.0330(0.0070), 1.0700(0.0010)]$} \\
\hline 1000 & {$[1.0575(0.0075), 1.0699(0.0007)]$} & {$[1.0546(0.0078), 1.0689(0.0008)]$} \\
\hline
\end{tabular}

Table 1: Lower and dual upper bounds with standard deviations for RMC and PRMC algorithms.

performs a bit worse than RMC (Regression MC), but the difference becomes smaller as $N_{\text {tr }}$ increases.

\section{Perturbation analysis for linear regression}

Consider a least squares problem of the form

$$
\beta^{\circ}=\underset{\beta \in \mathbb{R}^{d}}{\arg \min } \sum_{i=1}^{N}\left(Y_{i}-\beta^{\top} U_{i}\right)^{2},
$$

where for $i=1, \ldots, N,\left(Y_{i}, U_{i}\right)$ are i.i.d. pairs of a random variable $Y_{i}$ and a random (column) vector $U_{i} \in \mathbb{R}^{d}$. With $U:=\left(U_{1}, \ldots, U_{N}\right) \in \mathbb{R}^{d \times N}, Z=$ $N^{-1 / 2} U^{\top}$, and $V=N^{-1 / 2}\left(Y_{1}, \ldots, Y_{N}\right)^{\top}$, the solution of the problem (36) can be written in terms of pseudo inverses (denoted with $\dagger$ ),

$$
\beta^{\circ}=\left(U U^{\top}\right)^{-1} U Y=\left(Z^{\top} Z\right)^{-1} Z^{\top} V=Z^{\dagger} V .
$$


Consider now the least squares problem (36) due to a perturbation $\left(\widetilde{Y}_{i}, \widetilde{U}_{i}\right)$ of the pairs $\left(Y_{i}, U_{i}\right)$, and define $\widetilde{Z}$ and $\widetilde{V}$ accordingly. We so consider (cf. (37))

$$
\widetilde{\beta}^{\circ}=\left(\widetilde{Z}^{\top} \widetilde{Z}\right)^{-1} \widetilde{Z}^{\top} \widetilde{V}=\widetilde{Z}^{\dagger} \widetilde{V}
$$

and set

$$
\widetilde{Z}=Z+E, \quad \widetilde{V}=V+F .
$$

While the rows of $Z$ and the components of $V$ are independent, the rows of the perturbation matrix $E$ and the components of the perturbation vector $F$ are generally dependent. Also we note that we don't assume any kind of independence between the perturbations $E$ and $F$ and the matrix $Z$ and vector $V$, respectively.

Theorem 9 Consider the least squares problem (36) with solution (37), and its perturbation due to (39) with solution (38), respectively. Assume that $U_{1}, \ldots, U_{N}$ in (36) are i.i.d. random vectors in $\mathbb{R}^{d}$ such that for some $M>0,\left\|U_{1}\right\| \leq M$ a.s. Set

$$
\mathrm{E}\left[U_{1} U_{1}^{\top}\right]=\Sigma,
$$

so that

$$
Z^{\top} Z=\frac{1}{N} U U^{\top}=\frac{1}{N} \sum_{i=1}^{N} U_{i} U_{i}^{\top} .
$$

Let $\lambda_{\min }(\Sigma)$ be the smallest eigenvalue, and $\lambda_{\max }(\Sigma)$ be the largest eigenvalue of $\Sigma$, respectively. Then for any $\rho \in\left(0, \lambda_{\min }(\Sigma)\right)$ and $\varepsilon \in\left(0, \lambda_{\min }(\Sigma)-\rho\right)$ we have on the set $\mathcal{C}:=\mathcal{C}_{1} \cap \mathcal{C}_{2} \cap \mathcal{C}_{3} \cap \mathcal{C}_{4}$ with

$$
\begin{array}{ll}
\mathcal{C}_{1}: & \lambda_{\max }\left(Z^{\top} Z\right)<\lambda_{\max }(\Sigma)+\varepsilon, \\
\mathcal{C}_{2}: & \lambda_{\min }\left(Z^{\top} Z\right)>\lambda_{\min }(\Sigma)-\varepsilon, \\
\mathcal{C}_{3}: & \lambda_{\min }(\Sigma)-\left(2 \sqrt{\lambda_{\max }(\Sigma)+\varepsilon}+1\right)\|E\|>\rho+\varepsilon, \\
\mathcal{C}_{4}: & \|E\|<1 .
\end{array}
$$

that

$$
\left\|\widetilde{\beta}^{\circ}-\beta^{\circ}\right\| \leq c_{1}(\Sigma, \varepsilon, \rho)\|E\|\|V\|+c_{2}(\Sigma, \varepsilon, \rho)\|F\|,
$$

where

$$
\begin{aligned}
& c_{1}(\Sigma, \varepsilon, \rho):=\frac{1}{\rho}+\frac{2\left(\lambda_{\max }(\Sigma)+\varepsilon\right)+\sqrt{\lambda_{\max }(\Sigma)+\varepsilon}}{\rho^{2}} \text { and } \\
& c_{2}(\Sigma, \varepsilon, \rho):=c_{1}(\Sigma, \varepsilon, \rho)+\frac{\sqrt{\lambda_{\max }(\Sigma)+\varepsilon}}{\lambda_{\min }(\Sigma)-\varepsilon} .
\end{aligned}
$$

Furthermore, for any $\delta \in(0,1)$, and $N$ such that

$$
\varepsilon=\varepsilon_{\delta, N}=M \sqrt{\frac{\log (2 d / \delta)}{N C}} \frac{\lambda_{\max }^{3 / 2}(\Sigma)}{\lambda_{\min }(\Sigma)} \leq \lambda_{\min }(\Sigma)-\rho
$$


(cf. (51)), one has for the probability of $\mathcal{C}$,

$$
\mathrm{P}[\mathcal{C}] \geq 1-\delta-C_{p}\left(\left(\frac{2 \sqrt{\lambda_{\max }(\Sigma)+\varepsilon}+1}{\lambda_{\min }(\Sigma)-\varepsilon-\rho}\right)^{p}+1\right),
$$

provided $\delta$ and $C_{p}:=\mathrm{E}\left[|E|^{p}\right]$ are small enough (such that the above bound is positive).

Proof. Note that $\mathcal{C}:=\mathcal{C}_{1} \cap \mathcal{C}_{2} \cap \mathcal{C}_{3} \cap \mathcal{C}_{4}$ implies (46) in Lemma 11 and so by this Lemma,

$$
\begin{aligned}
\left\|(Z+E)^{\dagger}-Z^{\dagger}\right\| & \leq \frac{\|E\|}{\rho}\left[1+\frac{(2\|Z\|+1)\|Z\|}{\rho}\right] \\
& \leq \frac{\|E\|}{\rho}\left[1+\frac{2\left(\lambda_{\max }(\Sigma)+\varepsilon\right)+\sqrt{\lambda_{\max }(\Sigma)+\varepsilon}}{\rho}\right] \\
& =c_{1}(\Sigma, \varepsilon, \rho)\|E\|
\end{aligned}
$$

Thus, on $\mathcal{C}$ one has also,

$$
\begin{aligned}
\left\|(Z+E)^{\dagger}\right\| & \leq\left\|(Z+E)^{\dagger}-Z^{\dagger}\right\|+\left\|Z^{\dagger}\right\| \\
& \leq c_{1}(\Sigma, \varepsilon, \rho)+\frac{\sqrt{\lambda_{\max }(\Sigma)+\varepsilon}}{\lambda_{\min }(\Sigma)-\varepsilon}=c_{2}(\Sigma, \varepsilon, \rho),
\end{aligned}
$$

using that $\left\|Z^{\dagger}\right\| \leq\left\|\left(Z^{\top} Z\right)^{-1}\right\|\|Z\|$. So on $\mathcal{C}$ we get,

$$
\begin{aligned}
\left\|\widetilde{\beta}^{\circ}-\beta^{\circ}\right\| & =\left\|\widetilde{Z}^{\dagger} \widetilde{V}-Z^{\dagger} V\right\|=\left\|(Z+E)^{\dagger}(V+F)-Z^{\dagger} V\right\| \\
& \leq c_{1}(\Sigma, \varepsilon, \rho)\|E\|\|V\|+c_{2}(\Sigma, \varepsilon, \rho)\|F\| .
\end{aligned}
$$

For the probability of $\mathcal{C}$ one has

$$
\mathrm{P}[\mathcal{C}] \geq 1-\mathrm{P}\left[\Omega \backslash \mathcal{C}_{1} \cup \Omega \backslash \mathcal{C}_{2}\right]-\mathrm{P}\left[\Omega \backslash \mathcal{C}_{3}\right]-\mathrm{P}\left[\Omega \backslash \mathcal{C}_{4}\right]
$$

For the term $\mathrm{P}\left[\Omega \backslash \mathcal{C}_{1} \cup \Omega \backslash \mathcal{C}_{2}\right]$ we are going to apply Lemma 12. It is easy to see that, since $0<\lambda_{\min }(\Sigma) \leq \lambda_{\max }(\Sigma)$, (40) implies (52) in Lemma 12, So, due to this lemma, we have that

$$
\mathrm{P}\left[\Omega \backslash \mathcal{C}_{1} \cup \Omega \backslash \mathcal{C}_{2}\right] \leq \delta
$$

Furthermore,

$$
\begin{aligned}
\mathrm{P}\left[\Omega \backslash \mathcal{C}_{3}\right] & =\mathrm{P}\left[\lambda_{\min }(\Sigma)-\left(2 \sqrt{\lambda_{\max }(\Sigma)+\varepsilon}+1\right)\|E\| \leq \rho+\varepsilon\right] \\
& =\mathrm{P}\left[\frac{\lambda_{\min }(\Sigma)-\varepsilon-\rho}{2 \sqrt{\lambda_{\max }(\Sigma)+\varepsilon}+1} \leq\|E\|\right] \\
& \leq\left(\frac{2 \sqrt{\lambda_{\max }(\Sigma)+\varepsilon}+1}{\lambda_{\min }(\Sigma)-\varepsilon-\rho}\right)^{p} \mathrm{E}\left[\|E\|^{p}\right],
\end{aligned}
$$


and

$$
\mathrm{P}\left[\Omega \backslash \mathcal{C}_{4}\right]=\mathrm{P}[\|E\| \geq 1] \leq \mathrm{E}\left[\|E\|^{p}\right] .
$$

The statement now follows from (41).

Corollary 10 Let us take $\varepsilon=\rho=\lambda_{\min }(\Sigma) / 4$. Then with

$$
\begin{aligned}
c_{1}(\Sigma) & :=\frac{1}{\lambda_{\min }(\Sigma) / 4} \\
& +\frac{2\left(\lambda_{\max }(\Sigma)+\lambda_{\min }(\Sigma) / 4\right)+\sqrt{\lambda_{\max }(\Sigma)+\lambda_{\min }(\Sigma) / 4}}{\left(\lambda_{\min }(\Sigma) / 4\right)^{2}}, \\
c_{2}(\Sigma) & :=c_{1}(\Sigma)+\frac{\sqrt{\lambda_{\max }(\Sigma)+\lambda_{\min }(\Sigma) / 4}}{\lambda_{\min }(\Sigma)-\lambda_{\min }(\Sigma) / 4}
\end{aligned}
$$

we have on $\mathcal{C}$,

$$
\left\|\widetilde{\beta}^{\circ}-\beta^{\circ}\right\| \leq c_{1}(\Sigma)\|E\|\|V\|+c_{2}(\Sigma)\|F\|
$$

with probability

$$
\begin{aligned}
\mathrm{P}[\mathcal{C}] & \geq 1-2 d \exp \left[-N \frac{C \lambda_{\min }^{4}(\Sigma)}{16 M^{2} \lambda_{\max }^{3}(\Sigma)}\right] \\
& -C_{p}\left(\left(\frac{2 \sqrt{\lambda_{\max }(\Sigma)+\lambda_{\min }(\Sigma) / 4}+1}{\lambda_{\min }(\Sigma) / 2}\right)^{p}+1\right) .
\end{aligned}
$$

\section{Proofs}

\subsection{Proof of Theorem 4}

Let $\mathbf{X}_{t}=\left(X_{t}^{1}, \ldots, X_{t}^{N}\right)$ be a vector of i.i.d. copies of the exact solution to (9), and define for fixed $t$,

$$
w_{N}:=\underset{h \in \mathcal{H}_{K_{N}}}{\arg \min }\left\{\frac{1}{N} \sum_{i=1}^{N}\left(f\left(X_{T}^{i}\right)-h\left(X_{t}^{i}\right)\right)^{2}\right\} .
$$

Further let us denote by $V, \widetilde{V} \in \mathbb{R}^{N}$ the column vectors with coordinates

$$
V_{i}=\frac{f\left(X_{T}^{i}\right)}{\sqrt{N}}, \quad \widetilde{V}_{i}:=\frac{f\left(X_{T}^{i, N}\right)}{\sqrt{N}}, \quad i=1, \ldots, N,
$$

respectively, and consider the $\mathbb{R}^{N \times K}$ matrices

$$
\begin{aligned}
\widetilde{Z} & =\left(\psi_{k}\left(X_{t}^{i, N}\right) / \sqrt{N}, i=1, \ldots, N, k=1, \ldots, K\right), \\
Z & =\left(\psi_{k}\left(X_{t}^{i}\right) / \sqrt{N}, i=1, \ldots, N, k=1, \ldots, K\right),
\end{aligned}
$$


respectively. Then we have

$$
\widetilde{w}_{N}=\widetilde{\beta}_{N}^{\top} \boldsymbol{\psi}_{K}(\cdot), \quad \widetilde{\beta}_{N}=\left(\widetilde{Z}^{\top} \widetilde{Z}\right)^{-1} \widetilde{Z}^{\top} \widetilde{V}=\widetilde{Z}^{\dagger} \widetilde{V}
$$

and

$$
w_{N}=\beta_{N}^{\top} \boldsymbol{\psi}_{K}(\cdot), \quad \beta_{N}=\left(Z^{\top} Z\right)^{-1} Z^{\top} V=Z^{\dagger} V
$$

with $\boldsymbol{\psi}_{K}=\left(\psi_{1}, \ldots, \psi_{K}\right)^{\top}$. By using that

$$
\left|T_{M} \widetilde{w}^{N}(x)-T_{M} w^{N}(x)\right| \leq\left|\widetilde{w}^{N}(x)-w^{N}(x)\right|
$$

almost surely, one has for any event $\mathcal{C} \in \mathcal{F}$

$$
\begin{gathered}
\left(\mathrm{E}\left[\int\left(T_{M} \widetilde{w}^{N}(x)-w(x)\right)^{2} \mu_{t}(d x)\right]\right)^{1 / 2} \leq \\
\left(\mathrm{E}\left[\int 1_{\mathcal{C}}\left(\widetilde{w}^{N}(x)-w^{N}(x)\right)^{2} \mu_{t}(d x)\right]\right)^{1 / 2}+2 M_{f}(\mathrm{P}[\Omega \backslash \mathcal{C}])^{1 / 2} \\
+\left(\mathrm{E}\left[\int\left(T_{M} w^{N}(x)-w(x)\right)^{2} \mu_{t}(d x)\right]\right)^{1 / 2} \\
\leq M_{K} \sqrt{K}\left(\mathrm{E}\left[\left\|\widetilde{\beta}_{N}-\beta_{N}\right\|^{2} 1_{\mathcal{C}}\right]\right)^{1 / 2} \\
+\left(\mathrm{E}\left[\int\left(T_{M} w^{N}(x)-w(x)\right)^{2} \mu_{t}(d x)\right]\right)^{1 / 2}
\end{gathered}
$$

Set

$$
\begin{aligned}
& U=\left(\psi_{k}\left(X_{t}^{i}\right), i=1, \ldots, N, k=1, \ldots, K\right)^{\top} \in \mathbb{R}^{N \times K}, \\
& V=\left(\left(f\left(X_{t}^{i, N}\right) / \sqrt{N}, i=1, \ldots, N\right)^{\top} \in \mathbb{R}^{N},\right. \\
& E=\left(\left(\psi_{k}\left(X_{t}^{i, N}\right)-\psi_{k}\left(X_{t}^{i}\right)\right) / \sqrt{N}, i=1, \ldots, N, k=1, \ldots, K\right) \in \mathbb{R}^{N \times K}, \\
& F=\left(\left(f\left(X_{t}^{i, N}\right)-f\left(X_{t}^{i}\right)\right) / \sqrt{N}, i=1, \ldots, N\right)^{\top} \in \mathbb{R}^{N},
\end{aligned}
$$

then, with $\Sigma=\mathrm{E}\left[U U^{\top}\right], d=K$, and $\left\|U_{i}\right\| \leq \sqrt{K} M_{K}$, Corollary 10 implies

$$
\left\|\widetilde{\beta}^{\circ}-\beta^{\circ}\right\|^{2} \leq 2 c_{1}^{2} M_{f}^{2}\|E\|^{2}+2 c_{2}^{2}\|F\|^{2},
$$

on a set $\mathcal{C}$ with probability

$$
\begin{aligned}
\mathrm{P}[\mathcal{C}] & \geq 1-2 K \exp \left[-N \frac{C \varkappa_{\circ}^{4}}{16 K M_{K}^{2}\left(\varkappa^{\circ}\right)^{3}}\right] \\
& -\mathrm{E}\left[\|E\|^{p}\right]\left(\left(\frac{\sqrt{5 \varkappa^{\circ}}+1}{\varkappa_{\circ} / 2}\right)^{p}+1\right),
\end{aligned}
$$


where constants $c_{1}, c_{2}$ only depend on $\varkappa_{0}, \varkappa^{\circ}$. In particular we may take

$$
\begin{aligned}
& c_{1}:=\frac{44+8 \sqrt{5 / \varkappa^{\circ}}}{\varkappa_{0}}, \text { and } \\
& c_{2}:=d_{1}+\frac{2 \sqrt{5 \varkappa^{\circ}}}{3 \varkappa_{0}}=\frac{132+2 \sqrt{5 \varkappa^{\circ}}+24 \sqrt{5 / \varkappa^{\circ}}}{3 \varkappa_{0}} .
\end{aligned}
$$

As a consequence,

$$
\begin{aligned}
\mathrm{E}\left[\left\|\widetilde{\beta}_{N}-\beta_{N}\right\|^{2} 1_{\mathcal{C}}\right] & \leq 2 c_{1}^{2} M_{f}^{2} \mathrm{E}\left[\|E\|^{2}\right]+2 c_{2}^{2} \mathrm{E}\left[\|F\|^{2}\right] \\
& \leq 2 c_{1}^{2} M_{f}^{2}\left(\mathrm{E}\left[\frac{1}{N} \sum_{i=1}^{N} \sum_{k=1}^{K}\left(\psi_{k}\left(X_{t}^{i, N}\right)-\psi_{k}\left(X_{t}^{i}\right)\right)^{2}\right]\right) \\
& +2 c_{2}^{2} \mathrm{E}\left[\frac{1}{N} \sum_{i=1}^{N}\left(f\left(X_{t}^{i, N}\right)-f\left(X_{t}^{i}\right)\right)^{2}\right] \\
& \leq\left(2 c_{1}^{2} M_{f}^{2} \sum_{k=1}^{K} L_{k}^{2}+2 c_{2}^{2} L_{f}^{2}\right) \mathrm{E}\left[\left|X_{t}^{, N}-X_{t}\right|^{2}\right]
\end{aligned}
$$

We further have for $p \geq 2$,

$$
\begin{aligned}
\left(\mathrm{E}\left[|E|^{p}\right]\right)^{1 / p} & \leq\left(\mathrm{E}\left[\left(\frac{1}{N} \sum_{i=1}^{N} \sum_{k=1}^{K}\left(\psi_{k}\left(X_{t}^{i, N}\right)-\psi_{k}\left(X_{t}^{i}\right)\right)^{2}\right)^{p / 2}\right]\right)^{1 / p} \\
& =\left(\left(\mathrm{E}\left[\left(\frac{1}{N} \sum_{i=1}^{N} \sum_{k=1}^{K}\left(\psi_{k}\left(X_{t}^{i, N}\right)-\psi_{k}\left(X_{t}^{i}\right)\right)^{2}\right)^{p / 2}\right]\right)^{2 / p}\right)^{1 / 2} \\
& \leq\left(\frac{1}{N} \sum_{i=1}^{N} \sum_{k=1}^{K}\left(\mathrm{E}\left[\left(\psi_{k}\left(X_{t}^{i, N}\right)-\psi_{k}\left(X_{t}^{i}\right)\right)^{p}\right]\right)^{2 / p}\right)^{1 / 2} \\
& \leq\left(\frac{1}{N} \sum_{i=1}^{N} \sum_{k=1}^{K} L_{k}^{2}\left(\mathrm{E}\left[\left|X_{t}^{i, N}-X_{t}^{i}\right|^{p}\right]\right)^{2 / p}\right)^{1 / 2} \\
& =\sqrt{\sum_{k=1}^{K} L_{k}^{2}\left(\mathrm{E}\left[\left|X_{t}^{\cdot, N}-X_{t}^{\cdot}\right|^{p}\right]\right)^{1 / p} .}
\end{aligned}
$$

Combining the latter bounds with (11) and Theorem 11.3 from [12, and taking $p=2$ for simplicity, we get (using subadditivity of the squareroot) 


$$
\begin{gathered}
\left(\mathrm{E}\left[\int\left(T_{M} \widetilde{w}^{N}(x)-w(x)\right)^{2} \mu_{t}(d x)\right]\right)^{1 / 2} \leq \\
\leq M_{K} \sqrt{2 K}\left(c_{1} M_{f} \sqrt{\sum_{k=1}^{K} L_{k}^{2}}+c_{2} L_{f}\right) \frac{C_{2}}{\sqrt{N}} \\
+2 M_{f} \sqrt{2 K} \exp \left[-N \frac{C \varkappa_{\circ}^{4}}{32 K M_{K}^{2}\left(\varkappa^{\circ}\right)^{3}}\right] \\
2 M_{f} \sqrt{\sum_{k=1}^{K} L_{k}^{2}\left(\frac{\sqrt{5 \varkappa^{\circ}}+1}{\varkappa_{0} / 2}+1\right) \frac{C_{2}}{\sqrt{N}}} \\
+c_{3} M_{f} \frac{\sqrt{1+\log N} \sqrt{K}}{\sqrt{N}} \\
+c_{4} \inf _{h \in \mathcal{H}_{K}}\left(\int(h(x)-w(t, x))^{2} \mu_{t}(d x)\right)^{1 / 2}
\end{gathered}
$$

for universal constants $c_{3}, c_{4}$. Summarizing, and using (23), yields (22).

\subsection{Proof of Lemma 5}

Let us observe that for $j<\mathcal{J}$,

$$
\begin{aligned}
& g_{\tau_{j+1}^{*}}\left(Z_{\tau_{j+1}}\right)- g_{\tau_{N, j+1}}\left(Z_{\tau_{N, j+1}}\right)=\left(g_{j+1}\left(Z_{j+1}\right)-g_{\tau_{N, j+1}}\left(Z_{\tau_{N, j+1}}\right)\right) 1_{\left\{\tau_{j+1}^{*}=j+1, \tau_{N, j+1}>j+1\right\}} \\
&+\left(g_{\tau_{j+1}^{*}}\left(Z_{\tau_{j+1}^{*}}\right)-g_{j}\left(Z_{j}\right)\right) 1_{\left\{\tau_{j+1}^{*}>j+1, \tau_{N, j+1}=j+1\right\}} \\
&+\left(g_{\tau_{j+1}^{*}}\left(Z_{\tau_{j+1}^{*}}\right)-g_{\tau_{N, j+1}}\left(Z_{\tau_{N, j+1}}\right)\right) 1_{\left\{\tau_{j+1}^{*}>j+1, \tau_{N, j+1}>j+1\right\}} .
\end{aligned}
$$

By abbreviating temporarily in this proof $\mathrm{E}:=\mathrm{E}_{\mathcal{G}_{j+1}}$, and denoting $\mathcal{R}_{N, j}:=$ $\mathrm{E}\left[g_{\tau_{j+1}^{*}}\left(Z_{\tau_{j+1}^{*}}\right)-g_{\tau_{N, j+1}}\left(Z_{\tau_{N, j+1}}\right) \mid Z_{j}\right]$, we have $\mathcal{R}_{N, j} \geq 0$ almost surely, and

$$
\begin{aligned}
\mathcal{R}_{N, j} & =\mathrm{E}\left[\left(g_{j+1}\left(Z_{j+1}\right)-\mathrm{E}\left[g_{\tau_{N, j+2}}\left(Z_{\tau_{N, j+2}}\right) \mid Z_{j+1}\right]\right) 1_{\left\{\tau_{j+1}^{*}=j+1, \tau_{N, j+1}>j+1\right\}} \mid Z_{j}\right] \\
& +\mathrm{E}\left[\left(\mathrm{E}\left[g_{\tau_{j+2}^{*}}\left(Z_{\tau_{j+2}^{*}}\right) \mid Z_{j+1}\right]-g_{j+1}\left(Z_{j+1}\right)\right) 1_{\left\{\tau_{j+1}^{*}>j+1, \tau_{N, j+1}=j+1\right\}} \mid Z_{j}\right] \\
& +\mathrm{E}\left[\mathrm{E}\left[g_{\tau_{j+2}^{*}}\left(Z_{\tau_{j+2}^{*}}\right)-g_{\tau_{N, j+2}}\left(Z_{\tau_{N, j+2}}\right) \mid Z_{j+1}\right] 1_{\left\{\tau_{j+1}^{*}>j+1, \tau_{N, j+1}>j+1\right\}} \mid Z_{j}\right] \\
& =T_{1}+T_{2}+\mathrm{E}\left[\mathcal{R}_{N, j+1} 1_{\left\{\tau_{j+1}^{*}>j+1, \tau_{N, j+1}>j+1\right\}} \mid Z_{j}\right] .
\end{aligned}
$$

For $T_{1}$ we have

$$
\begin{aligned}
T_{1} & =\mathrm{E}\left[\left(g_{j+1}\left(Z_{j+1}\right)-\mathrm{E}\left[g_{\tau_{j+2}^{*}}\left(Z_{\tau_{j+2}^{*}}\right) \mid Z_{j+1}\right]\right) 1_{\left\{\tau_{j+1}^{*}=j+1, \tau_{N, j+1}>j+1\right\}} \mid Z_{j}\right] \\
& +\mathrm{E}\left[\left(\mathrm{E}\left[g_{\tau_{j+2}^{*}}\left(Z_{\tau_{j+2}^{*}}\right) \mid Z_{j+1}\right]-\mathrm{E}\left[g_{\tau_{N, j+2}}\left(Z_{\tau_{N, j+2}}\right) \mid Z_{j+1}\right]\right) 1_{\left\{\tau_{j+1}^{*}=j+1, \tau_{N, j+1}>j+1\right\}} \mid Z_{j}\right],
\end{aligned}
$$


and since

$$
\begin{aligned}
C_{N, j+1}\left(Z_{j+1}\right) & \geq g_{j+1}\left(Z_{j+1}\right) \geq \mathrm{E}\left[g_{\tau_{j+2}^{*}}\left(Z_{\tau_{j+2}^{*}}\right) \mid Z_{j+1}\right] \\
& =C_{j+1}^{*}\left(Z_{j+1}\right) \geq \mathrm{E}\left[g_{\tau_{N, j+2}}\left(Z_{\tau_{N, j+2}}\right) \mid Z_{j+1}\right]
\end{aligned}
$$

on $\left\{\tau_{j+1}^{*}=j+1, \tau_{N, j+1}>j+1\right\}$, we get

$$
\begin{aligned}
0 & \leq T_{1} \leq \mathrm{E}\left[\left(C_{N, j+1}\left(Z_{l+1}\right)-C_{j+1}^{*}\left(Z_{j+1}\right)\right) 1_{\left\{\tau_{j+1}^{*}=j+1, \tau_{N, j+1}>j+1\right\}} \mid Z_{j}\right] \\
& +\mathrm{E}\left[\mathcal{R}_{N, j+1} 1_{\left\{\tau_{j+1}^{*}=j+1, \tau_{N, j+1}>j+1\right\}} \mid Z_{j}\right] .
\end{aligned}
$$

Similarly, for $T_{2}$ we have

$$
0 \leq T_{2} \leq \mathrm{E}\left[\left(C_{j+1}^{*}\left(Z_{j+1}\right)-C_{N, j+1}\left(Z_{j+1}\right)\right) 1_{\left\{\tau_{j+1}^{*}>j+1, \tau_{N, j+1}=j+1\right\}} \mid Z_{j}\right] .
$$

Combining (42), (43), and (44), yields

$$
\mathcal{R}_{N, j} \leq \mathrm{E}\left[\left|C_{N, j+1}\left(Z_{j+1}\right)-C_{j+1}^{*}\left(Z_{j+1}\right)\right| \mid Z_{j}\right]+\mathrm{E}\left[\mathcal{R}_{N, j+1} \mid Z_{j}\right] .
$$

By straightforward induction, using the tower property and the final condition $\mathcal{R}_{N, \mathcal{J}-1}=0$, we so obtain

$$
0 \leq C_{j}^{*}\left(Z_{j}\right)-\bar{C}_{N, j}\left(Z_{j}\right) \leq \sum_{l=j+1}^{\mathcal{J}-1} \mathrm{E}\left[\mid C_{N, l}\left(Z_{l}\right)-C_{l}^{*}\left(Z_{l}\right) \| Z_{j}\right] .
$$

Taking on both sides the $L_{p}$-norm, applying the triangle inequality, and using that

$$
\mathrm{E}\left[\mathrm{E}\left[\left|C_{N, l}\left(Z_{j}\right)-C_{l}^{*}\left(Z_{l}\right)\right| \mid Z_{j}\right]^{p}\right] \leq \mathrm{E}\left[\left|C_{N, l}\left(Z_{l}\right)-C_{l}^{*}\left(Z_{l}\right)\right|^{p}\right],
$$

finally gives (25).

\subsection{Proof of Theorem 7}

Theorem 4 implies,

$$
\mathrm{E}_{\mathcal{G}_{j+1}}\left[\left\|C_{N, j}-\bar{C}_{N, j}\right\|_{L_{2}\left(\mu_{j}\right)}^{2}\right] \leq c_{1}^{2} \epsilon_{N, K}^{2}+c_{2}^{2} \inf _{h \in \mathcal{H}_{K}}\left\|\bar{C}_{N, j}(\cdot)-h\right\|_{L_{2}\left(\mu_{j}\right)}^{2},
$$

almost surely, for some $\eta, c_{1}, c_{2}>0$, which do not depend on $j, K$, and $N$. Hence, for the unconditional expectation we get,

$$
\mathrm{E}\left[\left\|C_{N, j}-\bar{C}_{N, j}\right\|_{L_{2}\left(\mu_{j}\right)}^{2}\right] \leq c_{1}^{2} \epsilon_{N, K}^{2}+c_{2}^{2} \inf _{h \in \mathcal{H}_{K}} \mathrm{E}\left[\left\|\bar{C}_{N, j}(\cdot)-h\right\|_{L_{2}\left(\mu_{j}\right)}^{2}\right]
$$

and so

$$
\left\|C_{N, j}-\bar{C}_{N, j}\right\|_{L_{2}\left(\mu_{j}, \mathbb{P}\right)} \leq c_{1} \epsilon_{N, K}+c_{2} \inf _{h \in \mathcal{H}_{K}}\left\|\bar{C}_{N, j}(\cdot)-h\right\|_{L_{2}\left(\mu_{j}, \mathbb{P}\right)} .
$$


By using (45) and the unconditional expectation applied to Lemma 5 with $p=2$, we get

$$
\begin{aligned}
\left\|C_{N, j}-C_{j}^{*}\right\|_{L_{2}\left(\mu_{j}, \mathbb{P}\right)} & \leq\left\|C_{N, j}-\bar{C}_{N, j}\right\|_{L_{2}\left(\mu_{j}, \mathbb{P}\right)}+\left\|\bar{C}_{N, j}-C_{j}^{*}\right\|_{L_{2}\left(\mu_{j}, \mathbb{P}\right)} \\
& \leq c_{1} \epsilon_{N, K}+c_{2} \inf _{h \in \mathcal{H}_{K}}\left\|C_{j}^{*}-h\right\|_{L_{2}\left(\mu_{j}\right)} \\
& +\left(1+c_{2}\right)\left\|\bar{C}_{N, j}-C_{j}^{*}\right\|_{L_{2}\left(\mu_{j}, \mathbb{P}\right)} \\
& \leq \Delta_{N, K}+\left(1+c_{2}\right) \sum_{l=j+1}^{\mathcal{J}}\left\|C_{N, l}-C_{l}^{*}\right\|_{L_{2}\left(\mu_{l}, \mathbb{P}\right)}
\end{aligned}
$$

with $c=\max \left\{c_{1}, c_{2}\right\}$. We prove the statement by induction. Suppose that the inequality (26) holds for $j=k$, then

$$
\begin{aligned}
\left\|C_{N, \mathcal{J}-k-1}-C_{\mathcal{J}-k-1}^{*}\right\|_{L_{2}\left(\mu_{\mathcal{J}-k-1}, \mathbb{P}\right)} & \leq \Delta_{N, K} \\
& +\left(1+c_{2}\right) \sum_{l=0}^{k}\left\|C_{N, \mathcal{J}-l}-C_{\mathcal{J}-l}^{*}\right\|_{L_{2}\left(\mu_{\mathcal{J}-l, \mathbb{P})}\right.}, \\
& \leq \Delta_{N, K}+\Delta_{N, K}(1+c) \sum_{l=0}^{k}(2+c)^{l} \\
& =\Delta_{N, K}\left(1+\left((2+c)^{k+1}-1\right)\right) \\
& =\Delta_{N, K}(2+c)^{k+1}
\end{aligned}
$$

and (26) holds also for $j=k+1$.

\section{Appendix}

In this section we present two auxiliary lemmas that were needed in Section 8

Lemma 11 Let $\rho>0$ and the matrix $Z \in \mathbb{R}^{N \times d}$ be of full rank with $N>d$. Let $Z$ and $E \in \mathbb{R}^{N \times d}$ be such that

$$
\lambda_{\min }\left(Z^{\top} Z\right)-(2\|Z\|+1)\|E\|>\rho, \quad\|E\|<1 .
$$

Then we have

$$
\left\|(Z+E)^{\dagger}-Z^{\dagger}\right\| \leq \frac{\|E\|}{\rho}\left[1+\frac{(2\|Z\|+1)\|Z\|}{\rho}\right] .
$$

Proof. Denote

$$
\Delta=Z^{\top} E+E^{\top} Z+E^{\top} E,
$$

then using the identity

$$
\begin{aligned}
\left((Z+E)^{\top}(Z+E)\right)^{-1}-\left(Z^{\top} Z\right)^{-1} & =-\left((Z+E)^{\top}(Z+E)\right)^{-1} \Delta\left(Z^{\top} Z\right)^{-1} \\
& =-\left(Z^{\top} Z+\Delta\right)^{-1} \Delta\left(Z^{\top} Z\right)^{-1}
\end{aligned}
$$


we derive

$$
\begin{aligned}
\|\left(\left((Z+E)^{\top}\right.\right. & \left.(Z+E))^{-1}-\left(Z^{\top} Z\right)^{-1}\right) Z^{\top} \| \\
& \leq\left\|\left(Z^{\top} Z+\Delta\right)^{-1}\right\|\left\|\left(Z^{\top} Z\right)^{-1}\right\|(2\|Z\|+1)\|E\|\|Z\| \\
& \leq \frac{(2\|Z\|+1)\|E\|\|Z\|}{\rho^{2}},
\end{aligned}
$$

since we have $\left\|\left(Z^{\top} Z\right)^{-1}\right\|=\lambda_{\min }^{-1}\left(Z^{\top} Z\right)<\rho^{-1}$ and

$$
\begin{aligned}
\lambda_{\min }\left(Z^{\top} Z+\Delta\right) & =\inf _{|x|=1} x^{\top}\left(Z^{\top} Z+\Delta\right) x \geq \inf _{|x|=1} x^{\top} Z^{\top} Z x+\inf _{|x|=1} x^{\top} \Delta x \\
& \geq \lambda_{\min }\left(Z^{\top} Z\right)-\|\Delta\| \geq \lambda_{\min }\left(Z^{\top} Z\right)-(2\|Z\|+1)\|E\| \\
& >\rho>0 .
\end{aligned}
$$

Analogously we have

$$
\left\|\left((Z+E)^{\top}(Z+E)\right)^{-1} E\right\|=\left\|\left(Z^{\top} Z+\Delta\right)^{-1} E\right\| \leq \frac{\|E\|}{\rho},
$$

and then (47) follows by (48), (49), and the triangle inequality.

Lemma 12 Let $X_{1}, \ldots, X_{N}$ be independent identically distributed random vectors in $\mathbb{R}^{d}$ such that

$$
\mathrm{E}\left[X_{1} X_{1}^{\top}\right]=\Sigma
$$

and for some $M>0,\left\|X_{1}\right\| \leq M$ almost surely. Then for all $\delta \in(0,1)$,

$$
\begin{aligned}
\mathbb{P}\left(\left\{\lambda_{\max }\left(\frac{1}{N} \sum_{i=1}^{N} X_{i} X_{i}^{\top}\right)\right.\right. & \left.>\lambda_{\max }(\Sigma)+\varepsilon_{\delta, N}\right\} \cup \\
& \left.\left\{\lambda_{\min }\left(\frac{1}{N} \sum_{i=1}^{N} X_{i} X_{i}^{\top}\right)<\lambda_{\min }(\Sigma)-\varepsilon_{\delta, N}\right\}\right) \leq \delta,
\end{aligned}
$$

where

$$
\varepsilon_{\delta, N}=M \sqrt{\frac{\log (2 d / \delta)}{N C}} \frac{\lambda_{\max }^{3 / 2}(\Sigma)}{\lambda_{\min }(\Sigma)}
$$

for some absolute constant $C>0$, provided $N$ is large enough such that

$$
M \sqrt{\frac{\log (2 d / \delta)}{N C}} \leq \lambda_{\max }^{1 / 2}(\Sigma) .
$$

Proof. For $z>0$ we have

$$
\begin{gathered}
\mathrm{P}\left[\lambda_{\max }\left(\frac{1}{N} \sum_{i=1}^{N} X_{i} X_{i}^{\top}\right)-\lambda_{\max }(\Sigma)>z\right] \\
\leq \mathrm{P}\left[\left\|\frac{1}{N} \sum_{i=1}^{N} X_{i} X_{i}^{\top}-\Sigma\right\|>z\right] .
\end{gathered}
$$


On the other hand, since for positive matrices $A, B$,

$$
\left|\lambda_{\min }(A)-\lambda_{\min }(B)\right| \leq\left\|B^{-1}\right\|\|B\|\|B-A\|,
$$

we have for $0<z<\lambda_{\min }(\Sigma)$,

$$
\begin{aligned}
& \mathrm{P}\left[\lambda_{\min }\left(\frac{1}{N} \sum_{i=1}^{N} X_{i} X_{i}^{\top}\right)<\lambda_{\min }(\Sigma)-z\right] \\
& \leq \mathrm{P}\left[\left\|\frac{1}{N} \sum_{i=1}^{N} X_{i} X_{i}^{\top}-\Sigma\right\|>z \frac{\lambda_{\min }(\Sigma)}{\lambda_{\max }(\Sigma)}\right] .
\end{aligned}
$$

Theorem 5.44 in [17 implies that for any $s>0$,

$$
\mathrm{P}\left[\left\|\frac{1}{N} \sum_{i=1}^{N} X_{i} X_{i}^{\top}-\Sigma\right\|>\max \left\{\|\Sigma\|^{1 / 2} \sqrt{\frac{s^{2} M^{2}}{N}}, \frac{s^{2} M^{2}}{N}\right\}\right] \leq d \cdot \exp \left(-C s^{2}\right),
$$

where $C$ is an absolute constant. For $N$ such that $s^{2} M / N \leq\|\Sigma\|$ and $s=$ $\sqrt{C^{-1} \log (2 d / \delta)}$, we so obtain

$$
\begin{gathered}
\mathrm{P}\left[\left\|\frac{1}{N} \sum_{i=1}^{N} X_{i} X_{i}^{\top}-\Sigma\right\|>M \sqrt{\frac{\|\Sigma\| \log (2 d / \delta)}{N C}}\right] \leq \delta / 2, \quad \text { for } N \text { such that } \\
M \sqrt{\frac{\log (2 d / \delta)}{N C}} \leq\|\Sigma\|^{1 / 2}=\lambda_{\max }^{1 / 2}(\Sigma) .
\end{gathered}
$$

Thus, (50) follows from (55) and taking $z=\varepsilon_{\delta, N}$ given by (51) in (53) and (54), respectively.

\section{References}

[1] Leif BG Andersen. A simple approach to the pricing of bermudan swaptions in the multi-factor libor market model. Journal of Computational Finance, 3:5-32, 1999.

[2] Fabio Antonelli, Arturo Kohatsu-Higa, et al. Rate of convergence of a particle method to the solution of the McKean-Vlasov equation. The Annals of Applied Probability, 12(2):423-476, 2002.

[3] Denis Belomestny. Pricing bermudan options by nonparametric regression: optimal rates of convergence for lower estimates. Finance and Stochastics, 15(4):655-683, 2011.

[4] Denis Belomestny, Anastasia Kolodko, and John Schoenmakers. Regression methods for stochastic control problems and their convergence analysis. SIAM J. Control Optim., 48(5):3562-3588, 2009/10. 
[5] Denis Belomestny and John Schoenmakers. Projected particle methods for solving mckean-vlasov stochastic differential equations. arXiv preprint arXiv:1708.08087, 2017.

[6] Denis Belomestny and John Schoenmakers. Advanced simulation-based methods for optimal stopping and control. Palgrave Macmillan, London, 2018. With applications in finance.

[7] Mark Broadie and Paul Glasserman. Pricing american-style securities using simulation. Journal of Economic Dynamics and Control, 21(8):1323-1352, 1997.

[8] Jacques F Carriere. Valuation of the early-exercise price for options using simulations and nonparametric regression. Insurance: mathematics and Economics, 19(1):19-30, 1996.

[9] Daniel Egloff et al. Monte carlo algorithms for optimal stopping and statistical learning. The Annals of Applied Probability, 15(2):1396-1432, 2005.

[10] TD Frank. Stochastic feedback, nonlinear families of markov processes, and nonlinear fokker-planck equations. Physica A: Statistical Mechanics and its Applications, 331(3):391-408, 2004.

[11] Paul Glasserman. Monte Carlo methods in financial engineering, volume 53. Springer Science \& Business Media, 2003.

[12] László Györfi, Michael Kohler, Adam Krzyżak, and Harro Walk. A distribution-free theory of nonparametric regression. Springer Series in Statistics. Springer-Verlag, New York, 2002.

[13] F.A. Longstaff and E.S. Schwartz. Valuing american options by simulation: a simple least-squares approach. Review of Financial Studies, 14(1):113$147,2001$.

[14] Huyên Pham and Xiaoli Wei. Dynamic programming for optimal control of stochastic mckean-vlasov dynamics. SIAM Journal on Control and Optimization, 55(2):1069-1101, 2017.

[15] G Szego. Orthogonal polynomials, vol. 23 of amer. In Math. Soc. Colloq. Publ., Amer. Math. Soc., Providence, RI, 1975.

[16] J. Tsitsiklis and B. Van Roy. Regression methods for pricing complex american style options. IEEE Trans. Neural. Net., 12(14):694-703, 2001.

[17] Roman Vershynin. Introduction to the non-asymptotic analysis of random matrices. In Compressed sensing, pages 210-268. Cambridge Univ. Press, Cambridge, 2012.

[18] Daniel Z Zanger. Quantitative error estimates for a least-squares monte carlo algorithm for american option pricing. Finance and Stochastics, 17(3):503-534, 2013. 